\title{
Hybrid carcinoma of the salivary gland: salivary duct adenocarcinoma adenoid cystic carcinoma
}

\author{
M L Snyder \& A F G Paulino \\ Department of Pathology, University of Michigan Hospitals, Ann Arbor, MI, USA
}

Date of submission 23 February 1999

Accepted for publication 19 April 1999

Snyder M L \& Paulino A F G

(1999) Histopathology 35, 380-383

\section{Hybrid carcinoma of the salivary gland: salivary duct adenocarcinoma adenoid cystic carcinoma}

Aims: Hybrid tumours of the salivary gland are rare neoplasms that have been described only in the parotid and palate. Their recognition is important particularly when the component tumours have different biological behaviours. The occurrence of a submandibular hybrid tumour has not been reported.

Methods and results: We describe a case of a 36-year-old woman with a hybrid carcinoma composed of salivary duct adenocarcinoma and adenoid cystic carcinoma of the right submandibular gland. There was no evidence of a pre-existing or concurrent pleomorphic adenoma. The presence of the two components was verified by differential immunohistochemical staining using a panel of cytokeratin, vimentin, smooth muscle actin and S100. The patient subsequently developed metastases to the pelvis, lumbar, vertebra and wrist. The clinical course in this patient was consistent with the behaviour of the salivary duct adenocarcinoma component.

Conclusions: The histogenesis of hybrid tumours is largely unknown, but in this case it may represent diverging differentiation of luminal tumour cells. Because some histological features of different salivary gland tumours overlap, immunohistochemistry is a valuable tool especially when used to delineate the components of a hybrid tumour.

Keywords: adenoid cystic carcinoma, hybrid tumour, salivary duct adenocarcinoma

\section{Introduction}

Hybrid carcinomas of the salivary gland are rare tumours that contain features of two separate and distinct tumour lines arising within the same lesion. Only three cases have been reported thus far in the English literature, two involving the palate and the other in the parotid. ${ }^{1,2}$ We describe the first case of hybrid carcinoma occurring in the submandibular gland in a 36-year-old woman. The tumour consisted of salivary duct carcinoma and adenoid cystic carcinoma, with the classic architectural components of each admixed, as seen histologically and confirmed immunohistochemically.

Address for correspondence: Dr A F G Paulino, Department of Pathology, University of Michigan Hospitals, 1500 East Medical Center Drive, Room 2G332, Ann Arbor, MI 48103, USA.

\section{Case report}

A 36-year-old woman consulted her primary physician because of a right submandibular mass that had been present for 1 year. During this time the patient noted a 40-pound weight loss but was otherwise asymptomatic. Apart from a 20-pack-year smoking history, her past medical history was unremarkable. Initial physical examination revealed no oropharyngeal lesions and no cervical adenopathy. She underwent excision of the mass. At the local hospital, the tumour was initially called an adenoid cystic carcinoma that had extended to the surgical margins. Re-excision revealed residual adenoid cystic carcinoma involving bone and soft tissue. Partial neck dissection revealed 13 lymph nodes negative for metastases. She was then referred to the University of Michigan Hospitals for further management. She received radiotherapy and on further imaging studies was found to have metastatic disease in the 
pelvis, lumbar region and wrist. The clinical suspicion of metastases were not confirmed histologically.

\section{PATHOLOGICAL FINDINGS}

The right submandibular gland taken during the initial resection measured $35 \times 25 \times 20 \mathrm{~mm}$ and was virtually completely replaced by tumour. The cut surface was hard and white. Microscopically various architectural patterns were observed. Peripherally, the classic cribriform pattern of adenoid cystic carcinoma was present (Figure 1). The tumour cells appeared basaloid, with angular nuclei, small nucleoli, scant eosinophilic cytoplasm and indistinct cell borders. The cyst-like spaces contained pale basophilic material as well as eosinophilic basal lamina material. About $80 \%$ of the tumour, however, consisted of salivary duct adenocarcinoma. There were solid nests of pleomorphic cells with central comedo-type necrosis and occasional dystrophic calcification (Figure 2). Mitotic figures were readily identified. In addition, small infiltrating cords and ductular structures within dense fibrosis (Figure 3) and perineural invasion were noted. No vascular invasion was identified. The tumour did not exhibit features of a preexisting or concurrent pleomorphic adenoma.

Sections of formalin-fixed, paraffin-embedded tissue were stained for high molecular weight cytokeratin (1:5 dilution, Sigma, St. Louis, MO), smooth muscle actin (1:1600, Sigma), vimentin (1:20, Dako, Carpinteria, CA), S100 (Dako), oestrogen receptor (ER) (predilute Ventana Medical Systems) and progesterone receptor (PR) (1:2, Abbott, Abbott Park, IL) and BRST-2 (1:50,

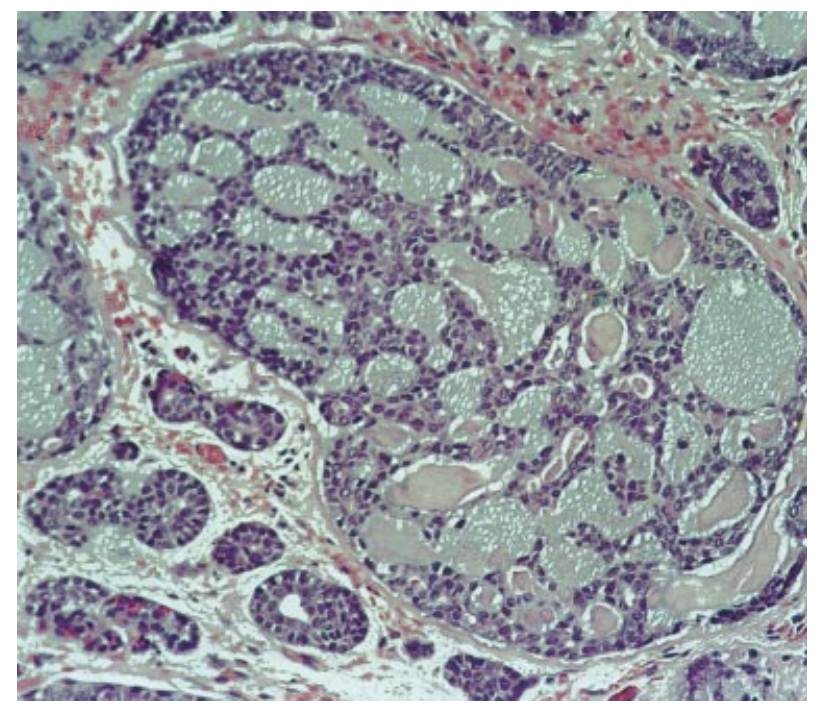

Figure 1. Classic cribriform pattern of adenoid cystic carcinoma.

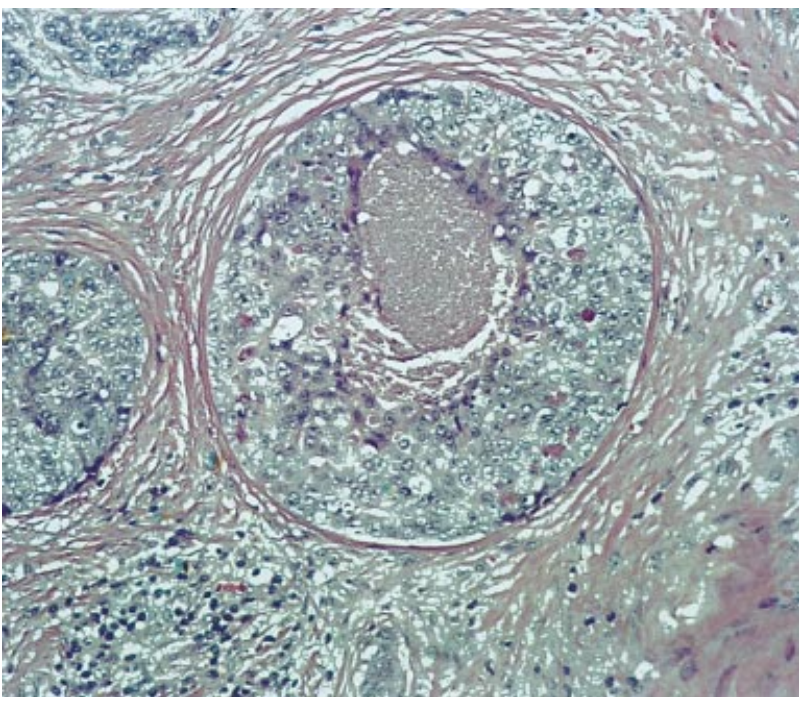

Figure 2. Salivary duct adenocarcinoma showing solid nests with central comedo-type necrosis.

Signet, Dedham, MA). The salivary duct carcinoma component was positive for cytokeratin and S100 but was negative for actin, ER/PR and BRST-2. The inner layer of the adenoid cystic carcinoma was positive for only cytokeratin. In contrast, the outer layer was negative for cytokeratin but positive for actin and vimentin.

\section{Discussion}

A hybrid tumour of the salivary gland is composed of two distinct tumour lines intimately admixed within

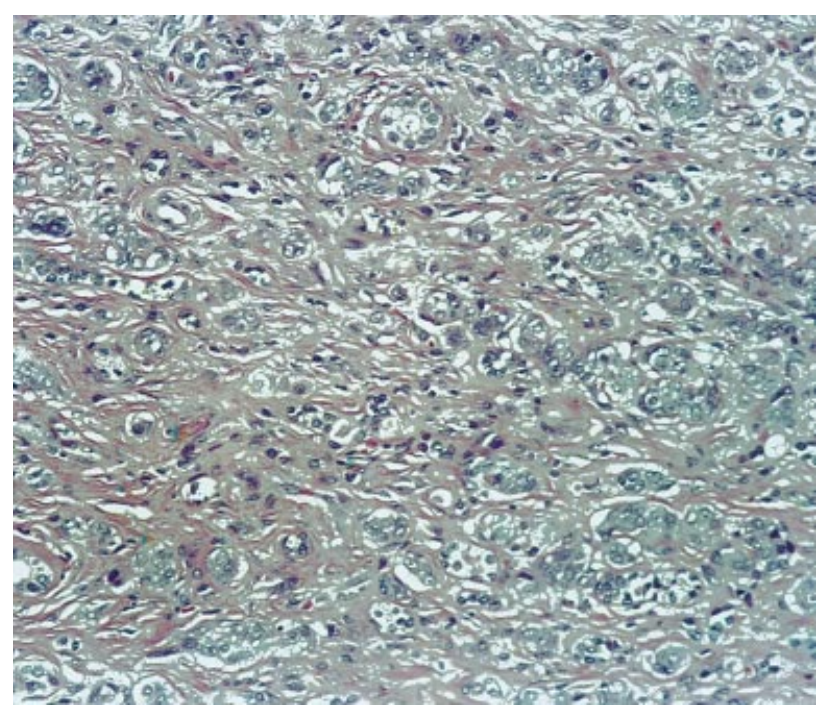

Figure 3. Salivary duct adenocarcinoma composed of small infiltrating cords and ductular structures within dense fibrosis. 
the same neoplasm. It must be differentiated from: (1) synchronous tumours which are composed of histologically distinct tumours presenting as separate nodules; ${ }^{3,4}(2)$ malignancies arising from a pre-existing benign tumour, as in carcinomas ex mixed tumour; or (3) collision tumours in which two malignant tumours abut each other but actually arise from separate primary sites.

Hybrid tumours of the salivary gland are rare neoplasms. Seifert reported five cases from over 6600 salivary gland tumours. ${ }^{1}$ The tumours arose in the parotid gland, except in one case which involved the palate. Three cases were malignant composed, respectively, of acinic cell carcinoma and salivary duct carcinoma; epithelial-myoepithelial carcinoma and adenoid cystic carcinoma; basal cell adenoma and adenoid cystic carcinoma. A hybrid carcinoma made up of adenoid cystic carcinoma and salivary duct adenocarcinoma has been documented in the palate. ${ }^{2}$ Our case had the same combination and similar immunohistochemical findings but occurred in the submandibular gland.

Salivary duct adenocarcinoma is a rare high-grade neoplasm most commonly arising in the parotid gland. Comedo-type necrosis is a common feature, with some histological and immunohistochemical similarities to ductal carcinoma of the breast. ${ }^{5}$ Salivary duct adenocarcinoma has an aggressive course with early, widespread metastases. ${ }^{6}$

Adenoid cystic carcinoma most often involves the parotid, submandibular gland and palate. ${ }^{7}$ As with most salivary gland neoplasms, the tumour is slightly more common in females than in males. Histologically, a mixture of cribriform, tubular and solid patterns may be seen. Some believe that adenoid cystic carcinoma with a solid growth pattern has a worse prognosis. ${ }^{8}$ However histological grading of adenoid cystic carcinoma is fraught with lack of reproducibility. ${ }^{9}$ Clinical stage appears to be the more significant prognostic factor rather than the histological subtype. ${ }^{10}$

Some features of adenoid cystic carcinoma and salivary duct carcinoma may overlap. Necrosis may occasionally be seen in the solid variant of adenoid cystic carcinoma. The tubular pattern of adenoid cystic carcinoma may simulate the infiltrating ductules of salivary duct carcinoma. Perineural invasion can be seen in both entities. Attention to cytological detail helps in distinguishing the two tumours. The cells of adenoid cystic carcinoma appear basaloid while those of salivary duct adenocarcinoma are more pleomorphic accompanied by mitotic figures. Confirmation of the two tumour lines in this case was made by using immunohistochemical stains for actin, cytokeratin, vimentin and S100. Expression of breast markers is variable in salivary duct carcinoma. ${ }^{5}$ Stains for oestrogen receptors, progesterone receptors and BRST-2 were negative in this case and were not useful as differential markers.

Differentiating the two tumours is prognostically important even if one believes that the solid variant of adenoid cystic carcinoma has a worse prognosis compared to the conventional cribriform subtype. The 5-year survival rate for salivary duct adenocarcinoma is $30 \%$, which is still more dismal than the $42 \% 5$-year survival rate for the solid form of adenoid cystic carcinoma. ${ }^{11,12}$

In conclusion, we report a hybrid carcinoma composed of salivary duct adenocarcinoma and adenoid cystic carcinoma. The origin of hybrid tumours is still largely unknown. In our case, it may represent diverging differentiation of luminal tumour cells, with one line exhibiting the features of salivary duct adenocarcinoma, while the other line the features of adenoid cystic carcinoma. The lineage of each was confirmed by immunohistochemistry. The behaviour of the tumour is most consistent with the salivary duct carcinoma component.

\section{References}

1. Seifert G, Donath K. Hybrid tumors of the salivary glands: definition and classification of five rare cases. Eur. J. Cancer B. Oral. Oncol. 1996; 32; 251-259.

2. Kamio N, Tanaka Y, Mukai M et al. A hybrid carcinoma: adenoid cystic carcinoma and salivary duct carcinoma of the salivary gland. Virchows Arch. 1998; 430; 495-500.

3. Misselevich I, Fradis M, Podoshin L, Boss J. Salivary gland double tumor: synchronous ipsilateral pleomorphic adenoma and acinic cell carcinoma of the parotid gland. Ann. Otol. Rhinol. Laryngol. 1997; 106; 226-229.

4. Gnepp DR, Schroeder W, Heffner D. Synchronous tumors arising in a single major salivary gland. Cancer 1989; 63; $1219-1224$.

5. Barnes L, Rao U, Contis L, Krause J, Schwartz A, Scalamogna P. Salivary duct carcinoma. Part II. Immunohistochemical evaluation of 13 cases for estrogen and progesterone receptors, cathepsin D and e-erbB-2 protein. Oral Surg. Oral Med. Oral Pathol. 1994; 78; 74-80.

6. Guzzo M, Di Palma S, Grandi C, Molinari R. Salivary duct carcinoma: clinical characterisitics and treatment strategies. Head Neck 1997; 19; 126-133.

7. Ellis GL, Auclair PL. Malignant epithelial tumors. In Rosai J ed. Tumor of Salivary Glands. Atlas of Tumor Pathology, Vol. 18. 3rd Series. Bethesda, Maryland: Armed Forces Institute of Pathology, 1995: 155-373.

8. Perzin KH, Gullane P, Clairmont AC. Adenoid cystic carcinoma of the salivary glands. A correlation of histologic features and clinical course. Cancer 1978; 42; 265-282.

9. Therkidlsen MH, Reibel J, Shiodt T. Observer variability in 
histological malignancy grading of adenoid cystic carcinomas. APMIS 1997; 105; 559-565.

10. Spiro RH, Huvos AG, Strong EW. Adenoid cystic carcinoma of salivary origin: a clinicopathological study of 242 cases. Am. J. Surg. 1974; 128; 512-520.

11. Jones AS, Hamilton JW, Rowley H, Husband D, Helliwell TR.
Adenoid cystic carcinoma of the head and neck. Clin. Otolaryngol. 1997; 22; 434-443.

12. Lewis JE, McKinney BC, Weiland LH, Ferreiro JA, Olsen KD. Salivary duct carcinoma-clinicopathologic and immunohistochemical review of 26 cases. Cancer 1996; 77; 223230.

(C) 1999 Blackwell Science Ltd, Histopathology, 35, 380-383. 